\title{
Demo Abstract: Inviz: Low-power Personalized Gesture Recognition Using Wearable Textile Capacitive Sensor Arrays
}

\author{
Gurashish Singh, Alexander Nelson, Ryan Robucci, Chintan Patel, Nilanjan Banerjee \\ Department of Computer Science and Electrical Engineering \\ University of Maryland, Baltimore County \\ \{singhg1, alnel1, robucci, cpatel2, nilanb\}@umbc.edu
}

\begin{abstract}
This demonstration presents Inviz, a low-cost gesture recognition system that uses flexible textile-based capacitive sensors. Gestures are recognized using proximity-based movement detection using flexible capacitive sensor arrays that can be built into the environment or placed on to the body or be integrated into clothing. Inviz provides an innovative interface to home automation systems to simplify environmental control for individuals with limited-mobility resulting from paralysis, paresis, and degenerative diseases. Proximity-based sensing obviates the need for physical contact which can result in skin abrasion which is particularly deleterious to people with limited-to-no sensitivity in their extremities. A custom-designed wireless module maintains a small form factor facilitating placement based on an individual's needs. Our system leverages a hierarchical sensing technique which facilitates learning gestures based on the individual and placement of the sensors. Classification uses just-in-time embedded computational resources to provide accurate responses while maintaining a low average power consumption, in turn reducing the impact of batteries on the form factor. To illustrate the use of Inviz in a smart home environment, we demonstrate an endto-end home automation system that controls small appliances. We will interface our system with a home automation gateway to demonstrate a subset of potential applications. This interactive demonstration highlights the intuitiveness and extensibility of the Inviz prototype.
\end{abstract}

\section{INTRODUCTION}

Approximately 1.5 million individuals in the United States are hospitalized each year because of strokes, brain injuries and spinal cord injuries [1], [2], [3]. Often these injuries cause severe impairments such as paralysis, paresis, weakness and limited range of motion. Extensive periods of expensive rehabilitation are necessary for individuals with such severe impairments because physical recovery can be a slow process [4], [5]. Assistive technologies can help reduce the time spent in rehabilitation by supplementing the direct care allowing the patients to become independent sooner.

Environment control is one of the keys to becoming independent while rehabilitation. Gesture recognition systems are capable of allowing patients with mobility impairments greater control over their environment. Several techniques such as the use of inertial sensors, vision systems, and other forms of tracking can be used to capture body gestures [6], [7], [8], [9], [10]. Gesture recognition systems for individuals with mobility impairments, however, present a set of fundamental challenges that typical gesture recognition systems often fail to address. First, sensors for gesture recognition are intrusive,

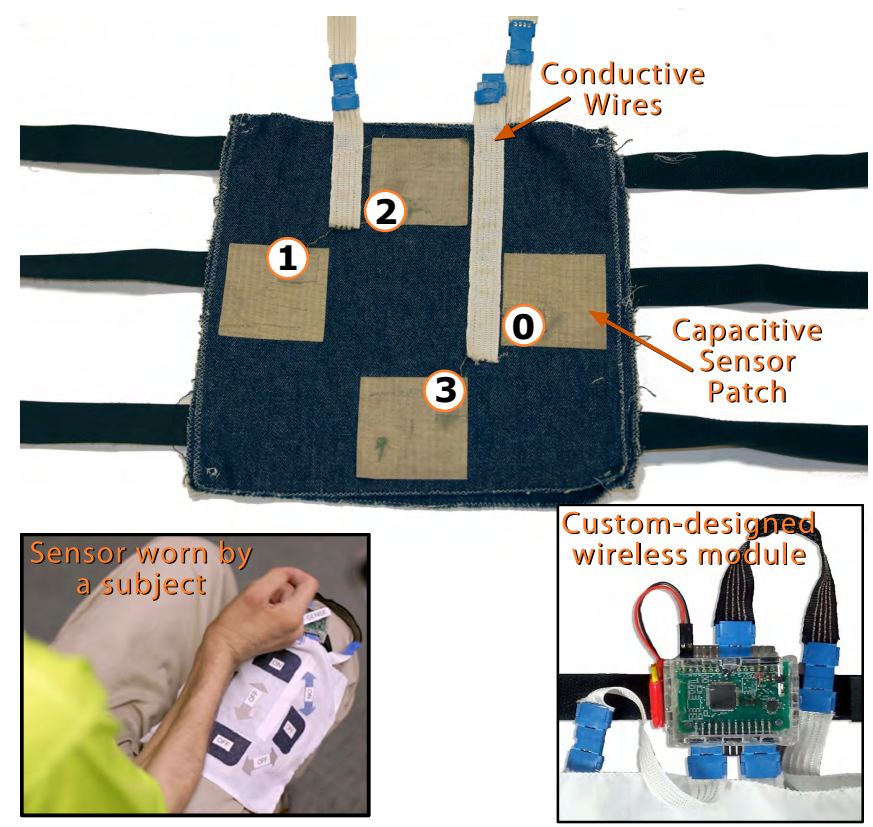

Fig. 1. The figure shows a prototype Inviz system worn by a patient with a spinal cord injury. The top figure demonstrates the capacitive sensor array sewn into the denim fabric using conductive wires. The data from the sensors is analyzed using our custom-designed wireless module which uses capacitance measurement ICs, an MSP430 micro-controller, and Bluetooth Low Energy (BLE) wireless module.

bulky, or expensive [11]. Eye tracking systems necessitate the use of mounted cameras while evoked-potential or touchbased systems use electrodes that can cause skin irritation and abrasion, conditions that can have a deleterious effect if unnoticed due to diminished sensation in the extremities. Second, existing systems are often not suitable for mobility impairments as they assume certain motions which a person may not be able to complete. There is a need, therefore, of systems that require minimal set-up and maintenance, and cause minimal fatigue and intrusiveness.

The above challenges are addressed by Inviz [12] which uses wearable sensors built from textile-based capacitivesensor arrays (CSA). These CSAs work on the principle of change in capacitance when there is movement in the proximity of the fabric capacitor plates. These plates can be sewn into clothing fabrics. Figure 1 illustrates a prototype Inviz system built using the capacitive plates and conductive threads sewn into the denim fabric. We have designed a low-power 


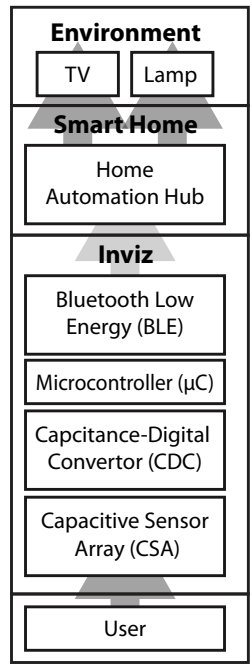

(a)

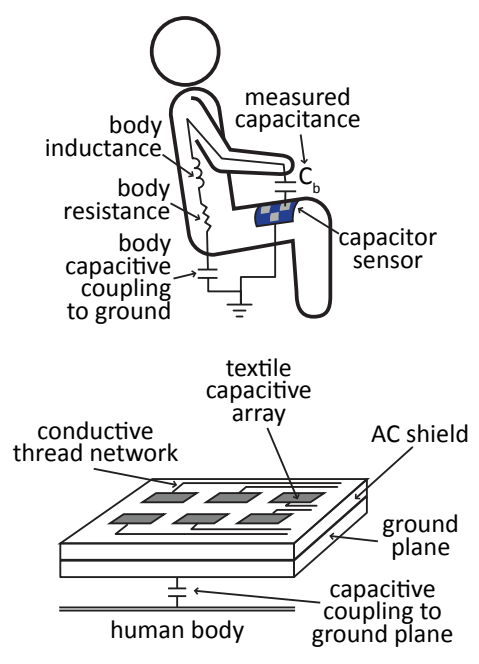

(b)
Fig. 2. (a) The figure illustrates our end-to-end home automation system. It demonstrates the data flow from the user to the environment through our system. (b) The top figure shows the equivalent electrical circuit when a capacitive plate is placed on the leg and the user performs gestures using his hand. The body is capacitively coupled to the sensor ground using a sensor ground plane. The bottom figure illustrates a longitudinal cross sectional view of the sensor. The CSA is sewn into fabric. Data from the capacitive plates are collected using a network of conductive threads sewn into the fabric. The sensor array consists of two additional layers an AC shield layer and a ground plane that comprise of conductive textile plates sewn into fabric.

hierarchical signal processing algorithm that converts signals from a CSA to reliable gestures. Our prototype then uses these gestures to control appliances in the home. In Inviz, we support two broad categories of gestures (1) Swipes: moving the hand from one plate to another; and (2) Hovers: protruding the hand over a textile sensor plate and then retracting the hand from the plate.

\section{DEMONSTRATION}

During the demonstration session, we will present a fully functional prototype of Inviz as an end-to-end cyber-physical system for home automation. The system will be interfaced with a home automation hub to allow control over small household appliances as illustrated in Figure 2(a).

Practical Setup: Gestures recognized by the Inviz system are transmitted to a personal computer over BLE, and the PC device then controls appliances over Zwave connection using a Micasaverde Vera gateway. The Inviz prototype consists of a custom-designed PCB board with the capacitance measurement circuit, observation calculation and thresholding circuit (built into the capacitance measurement IC), an MSP430 micro-controller, and a BLE wireless module. The capacitive sensor plates were sewn into the denim fabric and attached to the data collection module using 4-ply conductive thread network as shown in Figure 2(b) with a linear resistance of $50 \Omega /$ meter. In our prototype implementation, we faced two challenges unique to designing textile-based wearable plates. First, the conductive threads are built by weaving silver-plated threads and non-conductive threads. Unfortunately, this leads to fraying on the ends of the thread and can cause microscopic shorts between adjacent threads which are difficult to diagnose, especially when vampire connectors were used to connect the thread to the data collection board. The second challenge was soldering onto the conductive threads which we mitigated using vampire FCC connectors.

Technical Requirements: The system requires the components of the prototype described above. Given the hardware requirements and a minimal setup of the $\mathrm{PC}$ hardware, the Inviz prototype will recognize the gestures for which it has currently been trained. An offline training of the machine learning algorithm is required for a different customization or integration of the capacitive patches. Further research is being conducted to automatically adapt and retrain the system given a change in the prototype environment.

\section{ACKNOWLEDGMENT}

This material is based upon work supported by the National Science Foundation under awards CNS-1305099 and IIS1406626, CNS-1308723, CNS-1314024, and the Microsoft SEIF Awards. Any opinions, findings, and conclusions or recommendations expressed in this material are those of the authors and do not necessarily reflect the views of the NSF or Microsoft.

\section{REFERENCES}

[1] Margaret Jean Hall, Shaleah Levant, and Carol J DeFrances. Hospitalization for stroke in us hospitals, 1989-2009. Diabetes, 18(23):23, 2012.

[2] Jean A Langlois, Wesley Rutland-Brown, and Marlena M Wald. The epidemiology and impact of traumatic brain injury: a brief overview. The Journal of head trauma rehabilitation, 21(5):375-378, 2006.

[3] National spinal cord injury statistical center.

[4] Sarah Steinbaum, J Duncan Harviel, Jonathan H Jaffin, and Marion H Jordan. Lightning strike to the head: case report. The Journal of Trauma and Acute Care Surgery, 36(1):113-115, 1994.

[5] Marilyn Seif Workinger and Ronald Netsell. Restoration of intelligible speech 13 years post-head injury. Brain Injury, 6(2):183-187, 1992.

[6] Holger Junker, Oliver Amft, Paul Lukowicz, and Gerhard Tröster. Gesture spotting with body-worn inertial sensors to detect user activities. Pattern Recognition, 41(6):2010-2024, 2008.

[7] Ari Y Benbasat and Joseph A Paradiso. An inertial measurement framework for gesture recognition and applications. In Gesture and Sign Language in Human-Computer Interaction, pages 9-20. Springer, 2002.

[8] Sung-Jung Cho, Jong Koo Oh, Won-Chul Bang, Wook Chang, Eunseok Choi, Yang Jing, Joonkee Cho, and Dong Yoon Kim. Magic wand: a hand-drawn gesture input device in 3-d space with inertial sensors. In IWFHR-9 2004, pages 106-111. IEEE, 2004.

[9] Ying Wu and Thomas S Huang. Vision-based gesture recognition: A review. In Gesture-based communication in human-computer interaction, pages 103-115. Springer, 1999.

[10] Yi Xia, Zhi-Ming Yao, Xian-Jun Yang, Sheng-Qiang Xu, Xu Zhou, and Yi-Ning Sun. A footprint tracking method for footprint tracking method for gait analysis. Biomedical Engineering: Applications, Basis and Communications, 26(01), 2014.

[11] Susan Koch Fager and Judith M Burnfield. Patients' experiences with technology during inpatient rehabilitation: opportunities to support independence and therapeutic engagement. Disability and Rehabilitation: Assistive Technology, 9(2):121-127, 2013.

[12] Gurashish Singh, Alexander Nelson, Ryan Robucci, Chintan Patel, and Nilanjan Banerjee. Inviz: Low-power personalized gesture recognition using wearable textile capacitive sensor arrays. In Perv. Comp. and Comm. (PerCom), IEEE Int. Conf. on. IEEE, 2015. 